\title{
The validation of the Italian Perceived Disability Scale (IPDS) in chronic daily headache sufferers
}

\author{
Marco Innamorati · Maurizio Pompili · Sergio De Filippis · Federica Gentili • \\ Denise Erbuto - David Lester - Antonino Tamburello - Giulia Iacorossi · \\ Ilaria Cuomo · Giovanni Dominici · Roberto Tatarelli $\cdot$ Paolo Martelletti
}

Received: 1 August 2008/Accepted: 16 September 2008/Published online: 15 October 2008

(C) Springer-Verlag 2008

\begin{abstract}
The goals of this study were to assess the validity and usefulness of a new scale and to assess disability in a sample of patients with chronic daily headache. Participants were a convenience sample of 61 adult outpatients admitted to the Department of Medical Sciences of the Sant'Andrea Hospital in Rome, between September 2007 and May 2008. Inclusion criteria were, a diagnosis of chronic daily headache (illness duration $>5$ years). Patients were administered the Beck Hopelessness Scale (BHS), a specific section of the Mini-International Neuropsychiatric Interview (MINI) assessing suicidal intent, the Gotland Male Depression Scale (GMDS), and a scale devised to measure the degree of disability caused by the headache [Italian Perceived Disability Scale (IPDS)]. Analyses
\end{abstract}

Electronic supplementary material The online version of this article (doi:10.1007/s10194-008-0076-0) contains supplementary material, which is available to authorized users.

M. Innamorati · A. Tamburello

Università Europea di Roma, Rome, Italy

M. Pompili $(\bowtie) \cdot$ F. Gentili · D. Erbuto - G. Iacorossi ·

I. Cuomo · G. Dominici · R. Tatarelli

Department of Psychiatry, Sant'Andrea Hospital,

Sapienza University of Rome, 1035 Via di Grottarossa, 00189 Rome, Italy

e-mail: maurizio.pompili@uniroma1.it;

mpompili@mclean.harvard.edu

M. Pompili

Harvard Medical School, McLean Hospital, Boston, USA

S. De Filippis · P. Martelletti

Department of Medical Sciences, Sant'Andrea Hospital,

Sapienza University of Rome, Rome, Italy

D. Lester

The Richard Stockton College of New Jersey, Pomona, NJ, USA indicated that the IPDS had good internal consistency (Cronbach alpha $=0.93$; average inter-item correlation $=0.40$ ), and good convergent validity, with moderate to strong associations with measures assessing emotional distress (BHS, $r=0.47$; $P<0.0001$; GMDS, $r=0.72$; $P<0.001)$. A single-item, logistic regression analysis indicated that the IPDS is able to predict suicide intent (Wald $\chi^{2}=5.04 ; P<0.05$ ) in chronic daily headache patients. The IPDS is a brief instrument that is useful for comparisons with other chronic illnesses, and it may be used both for basic research and clinical applications when screening for comorbidity with emotional distress and disorders.

Keywords Chronic daily headache .

Self-injurious behavior · Disability · Depression . Hopelessness

\section{Introduction}

Chronic illness is a permanently altered health state, caused by a non-reversible pathological condition leaving a disability that cannot be cured by a short course of medical therapies [1]. Chronic illnesses are associated with increased needs, physical and emotional pain, and increased disability. Disability associated with chronic medical illness is a major heath issue. Disability has great impact on the quality of life of patients, and it is associated with considerable costs for the health care system. In the USA, the direct medical costs for persons with disability were estimated to be almost $\$ 250$ billion each year [2].

Headache is one of the most common disorders encountered in pediatric and adult neurology clinics [3, 4]. Although the prevalence rates may vary according to age, 
gender and race, some studies report that tension-type headache may affect more than $20 \%$ of individuals in the general population, and migraine headache around $18 \%$ [5]. Rasmussen [6, 7] indicated a lifetime prevalence for any kind of headache of $93 \%$ in men and $99 \%$ in women. In the elderly, the reported prevalence for headaches ranges from 5 to almost $50 \%$ partly due to the different definitions and diagnostic criteria used in the studies [8-10]. Headache can be either mild and at infrequent intervals, or severe and enduring. The International Classification of Headache Disorders, II version (ICHD-II) recognizes 24 types of chronic headache and defines primary episodic headaches as chronic when the attacks appear for more than 15 days per month and for at least 3 months [11].

Headache is associated with emotional and economic costs [12-14]. Elston Lafata and colleagues [13] indicated that migraine patients have significantly higher medical care expenditures than nonmigraine patients even after controlling for the patients' sociodemographic variables and health insurance coverage. Once these factors are controlled, the average migraine patient is expected to incur $\$ 2,636$ in annual medical expenditures, while on average those not suffering from migraine are expected to incur annual medical expenditures of only $\$ 2,148$. Furthermore, headaches cause considerable disability in everyday activities, and a positive relationship has been reported between depression, anxiety, and disability in headache patients [15-18]. For example, Jelinski and colleagues [16], using a multivariable logistic regression model, reported that individuals with severe headacherelated disability were 1.6 times $(95 \% \mathrm{CI}, 1.03-2.48)$ more likely to be depressed than patients with weak to moderate disability.

There has been a realization in recent years that the impact of medical illness on an individual's life cannot be measured by disease indices alone, and that self-reported measures such as pain severity and limitation of daily activity provide important assessments of such conditions. Thus, we need to measure how headaches affect the daily life of sufferers and also self-report measures to assess people's perception of illness, health, and life satisfaction. Beliefs about an illness may influence the level of interference in daily life from that illness and on people's decision to seek treatment for it. Negative beliefs about illness may be an important issue to address in order to reduce the impact of disease and encouraging appropriate management.

The use of headache impact or disability tools is increasingly being recommended as part of generalized headache management guidelines, and reduction in headache-related disability is now considered a major goal of migraine treatments $[19,20]$, even though the doctors generally "don't ask" and patients "don't tell" about their migraine disability [21]. Generic and headache-specific measures are used to assess disability among headache patients. Both types of measures have limitations and strengths. Generic measures are composed of items evaluating symptoms not specific to headache, and so they may be used for comparisons across different disorders. Headache specific scales are composed only of items assessing symptoms specific for headache, and so they cannot be used for comparisons across different disorders. Among the disease-specific measures, the Migraine Disability Assessment questionnaire (MIDAS), the Headache Impact Test (HIT), and the Headache Disability Inventory (HDI) are the most commonly used instruments.

The MIDAS is a seven-item, self-administered questionnaire that sums the first five items, assessing the number of productive days lost over the past 3 months in three settings: school or paid work, household work, and non-work activities. The time interval was chosen by the authors to balance the accuracy of self-reported information with the clinical relevance of the headache experience over time. Questions ask about either days with missed activity or days where productivity was reduced by at least half. The last two items assess headache frequency and intensity. Population-based studies conducted in the USA and the UK $[22,23]$ resulted in sufficiently good psychometric properties, with internal consistency and test-retest reliability higher than 0.80 [23]. The MIDAS also has been translated and validated in several languages [24-29].

However, several issues have been raised about the scale. For example, Pryse-Phillips [30] indicated limitations associated with (1) the choice to separate work, household work and leisure-social activities; (2) the lack of distinction among complete inability and 50\% reduction in ability to perform activities; and (3) the targeting of the more severely affected headache patients. Andrasik et al. [31] also indicated limitations related to the time interval chosen by authors, because headache characteristics vary from attack to attack among migraine suffers, and so an individual headache attack could cause different effects on disability. Therefore, headache-related disability as measured by the MIDAS might be different from the disability as measured by diary-based methods.

The internet HIT is an instrument to measure the impact that headache has on an individual's ability to function on the job, at home, at school and in social situations. The paper-based version of the instrument is known as the HIT6 , and is available in over 25 languages [32]. HIT-6 items, selected from an already-existing item pool of 54 items and from 35 items suggested by clinicians, cover 6 content categories including (pain severity, activity limitation, wish to lie down, tiredness, irritability, and loss of concentration during work or daily activities due to headaches) has been widely used in surveys of headache impact. Internal 
consistency, parallel forms reliability, and test-retest reliability estimates of the HIT- 6 were $0.89,0.90$, and 0.80 , respectively [33]. Studies on the HIT-6 translations indicated that most translations (Canadian English, French, Greek, Hungarian, UK English, Hebrew, Portuguese, German, Spanish, and Dutch) were comparable to US English [34]. The HIT-6 was also responsive to selfreported changes in headache impact [33]. However, while the HIT-6 is easy to complete, calculating the composite score is complicated, combining questions about headache frequency and intensity along with questions regarding disability [31].

The HDI is a 25 -item questionnaire that measures the functional and emotional impact of headache on everyday life. It was derived from patient perceptions of headacherelated disability and was based on existing scales. The HDI has good internal consistency (0.89), test-retest reliability, and construct validity $[35,36]$. However, like the HIT-6, the HDI takes some effort to score [31].

Analysis of the literature indicates that the way in which headache patients perceive their health and disability is associated with emotional disorders and poor health behavior. Several authors have suggested that headacherelated disability should be assessed and used as an outcome measure in treatments for headache. In recent years, some instruments have been constructed to assess perceived disability and changes over time. However, those scale lack adequate psychometric properties, such as adequate Cronbach alpha reliability. For example, Nunnally and Bernstein [37] criticized researchers who were satisfied with an alpha of 0.80 for indicating good reliability. This may be sufficient for basic, academic research, but in clinical practice, where decisions are made about individuals, more reliable instruments are required; 0.90 as the bare minimum and 0.95 as the golden standard for instruments.

The aim of the present study was to assess the validity and usefulness of a new measure to assess disability (see electronic supplementary material) in a sample of patients with chronic daily headache.

\section{Methods}

Study site and participants

Participants were a convenience sample of 61 adult outpatients ( 8 women, 53 men) selected from 85 consecutive patients who met inclusion criteria (response rate, $72 \%$ ) and who were admitted to the Department of Medical Sciences of the Sant'Andrea Hospital in Rome between September 2007 and May 2008. Inclusion criteria were, a diagnosis of chronic daily headache (illness duration $>5$ years), and an age $\geq 18$ years; exclusion criteria were, comorbidity with major disorders of the central nervous system (such as Parkinson disease, dementia or epilepsy), delirium and any condition affecting the patient's ability to complete the assessment, including refusal to give informed consent. The average age of the participants was 47.0 years $(\mathrm{SD}=11.5$; min./max., 25/75 years). All the participants were also affected by a second form of headache; the medication overuse headache. There were no differences between participants and patients excluded from the sample in sociodemographic variables and diagnosis.

Participants participated voluntarily in the study, and each subject provided written informed consent. The study protocol received ethics approval from the local research ethics review board.

\section{Data collection and measurements}

Patients were administered the Beck Hopelessness Scale (BHS) [38], a specific section of the Mini-International Neuropsychiatric Interview (MINI) assessing suicidal intent [39], the Gotland Male Depression Scale (GMDS) [40, 41], and the new Italian Perceived Disability Scale (IPDS) to measure headache-related disability.

The BHS is a 20-item scale for measuring the cognitive component of the syndrome of depression. Hopelessness is a cognitive trait considered to be associated with suicide, leading suicidal patients to believe that suicide is the only feasible strategy for dealing with their seemingly insoluble problems. Empirical research has demonstrated a relationship between hopelessness and suicidal intent [42-44]. Beck et al. [45, 46] carried out two prospective studies of inpatient and outpatient samples. The authors found that hopelessness scores were related significantly to eventual completed suicide. A cutoff score of 9 or above identified most of the patients who eventually committed suicide. The high-risk group identified in the latter study was 11 times more likely to commit suicide than the rest of the outpatients. Thus, hopelessness may be used as an indicator of suicide potential. Italian validation of the BHS was provided by Pompili et al. [47], who confirmed the strong association between hopelessness and suicide risk.

The MINI, a DSM-IV-TR based short structured interview, was used to evaluate suicidal intent. The section assessing suicide intent evaluates past and current suicidal behavior and ideation and classifies subjects into four groups: no suicidal risk, low suicidal risk, moderate suicidal risk, and high suicidal risk. For the present study, subjects were classified into two groups: lower suicidal intent (no suicidal intent and low suicidal intent as measured by the MINI) and higher suicidal intent (moderate suicidal intent and high suicidal intent as measured by the MINI). 
The GMDS is a 13-item scale for measuring depression severity. Each item is rated on a Likert-type scale ranging from 0 to 3 . The raw score can vary between 0 and 39 . Scores below 13 mean the absence of clinical symptoms of depression, scores between 13 and 25 mean a probable presence of clinical depression, and scores higher than 25 mean clinical depression. The GMDS has good validity [48].

The IPDS is composed of 20 items, devised from one of the authors (M. I.), and measured on a 5-point Likert-type scale (completely false to completely true), assessing people's beliefs regarding autonomy/disability in different situations of life (for e.g., "my body is weak and unreliable"; "I will have to worry about my health conditions all my life long"; "I boil over more easier than in the past"). Explicit time limits were avoided, and the items were written using the present tense. To increase the reliability, scoring rules were made as explicit as possible. For example, the individual is instructed to use both frequency ("I never think this way", "I usually think this way") and intensity ("I rarely think this way, and it is easy to me to think that the situation different") criteria to rate each item. The raw score ranges from 0 to 80 .

\section{Data analysis}

To analyze items, we calculated the discrimination index (the corrected item-total correlation index); a discrimination index less than 0.2 mean the item is troublesome and should be excluded from the measure. Homogeneity was calculated using Cronbach alpha $(\alpha)$ and the average interitem correlation index. Correlation coefficients between the IPDS, the GMDS, and the BHS were calculated to assess convergent validity of the new measure with well validated instruments for measuring emotional distress. The mean score and standard deviation (SD) of the measure were also calculated. A univariate general linear model analysis was performed to assess the effects of sex, age, and their interaction on IPDS raw score. $t$ tests and single-variable logistic regression analysis with the MINI-based suicidal intent groups as the dependent variable were performed to assess usefulness of the IPDS in predicting suicidal intent in chronic daily headache patients.

All statistical analyses were performed using the SPSS 13.0 statistical software package.

\section{Results}

The analysis of the individual items of the IPDS indicated that all discrimination indices were above 0.40 ( $\mathrm{min} . / \mathrm{max}$., $0.54 / 0.87$ ), indicating a strong convergence but not redundancy of any single item.
The average raw score of the IPDS in the sample was 28.74 (SD, 17.23; min./max, 0-77). A univariate general linear model did not indicate a significant effect of either age $[F(d f: 2)=0.14 ; P=0.87]$, sex $[F(d f: 1)=1.80 ; P=$ $0.19]$, or their interaction $[F(d f: 1)=0.03 ; P=0.86]$ on the IPDS raw score. Only one patient scored 0 on the measure, while none scored 80, and so a "roof effect" was not evident, and floor effect was weak among this sample of headache patients. Cronbach $\alpha$ was 0.93 , and average interitem correlation was 0.40 , indicating good homogeneity for the scale items.

The convergent validity of the IPDS was satisfactory, with moderate to strong associations with measures assessing emotional distress (BHS, $r=0.47 ; P<0.001$. GMDS, $r=0.72 ; P<0.001)$. All the measures were significantly associated with suicidal intent. The patients with higher suicidal intent had an average IPDS raw score of $50.00(\mathrm{SD}=10.80)$ versus $24.37(\mathrm{SD}=15.95)$ for the patients with lower suicidal intent $[t(d f: 37)=-3.11$; $P<0.01], 17.75(\mathrm{SD}=9.00)$ versus $10.59(\mathrm{SD}=5.91)$ on the GMDS $[t(d f: 36)=-2.18 ; P<0.05]$, and 12.25 $(\mathrm{SD}=5.12)$ versus $6.09(\mathrm{SD}=4.17)$ on the GMDS $[t(d f$ : $37)=-2.75 ; P<0.01]$. A single-item logistic regression analysis resulted in a significant model $\left(\chi^{2}=7.74\right.$; $P<0.01)$ explaining $37 \%$ of the variability of suicidal intent (Nagelkerke $R^{2}=0.37$ ). Thus, the IPDS contributed to the prediction of suicide intent (Wald $\chi^{2}=5.04$; $P<0.05)$. Chronic daily headache patients with higher suicidal intent were 1.10 times (95\% CI, 1.01/1.19) more at risk to have higher perceived disability on the IPDS.

\section{Discussion}

The aim of the present study was to asses the validity and usefulness of a new measure for assessing disability in a sample of patients with chronic daily headache. The Italian Perceived Disability Scale (IPDS) is composed of 20 items measured on a 5-point Likert-type scale, assessing people's beliefs regarding autonomy/disability in different situations of life. Our findings indicate that the IPDS is an easy to administer, self-report scale, useful for assessing disability in chronic daily headache patients. The strengths of our scale are: (1) the IPDS is not specific for headache-induced disability and so it may be useful for comparison of chronic headache with other chronic illnesses; (2) the administration and scoring of the IPDS are quick and intuitive, and it may be a useful instrument even by general practitioners; (3) the psychometric properties are good, both for basic research and for clinical applications, approximating the golden standard indicated by Nunnally and Bernstein [37]; and (4) the IPDS has a strong convergence, although it is not redundant, with measures assessing depression. 
Our results are also consistent with research indicating a positive relationship between disability and emotional distress in chronic daily headache patients [15-18, 49]. Thus, the IPDS may also be useful in treatment planning because treatments for headache will have to be tested for their effects on comorbid conditions. For example, in patients with comorbid major depression, it is important to avoid migraine treatments, such as beta-blockers, which produce side effects such as drowsiness, fatigue, lethargy, sleep disorders, nightmares, depression, memory disturbance and hallucinations [50]. This runs the risk of increasing the severity of the depressive symptoms. Instead, tricyclic antidepressants should be used, which would treat both the migraine and the depression.

Some earlier studies investigated the relationship among suicidal intent and headaches. For example, Breslau and Davis [51] reported that people with migraines had a higher lifetime rate of suicide attempts than those with no history of migraine. In addition, those with major depressive disorder (MDD) and migraines had higher rate of suicide attempts than those with only MDD. Breslau [52] found an association of migraines with aura with suicide attempts not necessarily due to coexisting MDD. Thus, the ability of the IPDS to predict suicidal intent in chronic daily headache patients is a considerable strength of this measure when used in screening programs to identify comorbidity with emotional distress and disorders.

The present study did have some limitations. First, we did not assess the convergent validity of the IPDS neither with diary-based measures, considered the golden standard for the assessment of disability in headaches, nor with headache-specific measures such as the MIDAS. Second, we did not evaluate the test-retest stability of the IPDS in this population.

In conclusion, self-report measures evaluating perceived disability may be important in headache management, and the IPDS may be a useful instrument in clinical settings when screening for comorbidity with emotional distress and disorders.

\section{Conflict of interest None.}

\section{References}

1. Miller J (1992) Coping with chronic illness: overcoming powerlessness. Davis, Philadelphia

2. (2001) Prevalence of disabilities and associated health conditions among adults-United States, 1999. MMWR Morb Mortal Wkly Rep 50:120-125

3. Linet MS, Celentano DD, Stewart WF (1991) Headache characteristics associated with physician consultation: a populationbased survey. Am J Prev Med 7:40-46
4. Pascual J, Combarros O, Leno C et al (1995) Distribution of headache by diagnosis as the reason for neurologic consultation. Med Clin (Barc) 104:161-164

5. Kurt S, Kaplan Y (2008) Epidemiological and clinical characteristics of headache in university students. Clin Neurol Neurosurg 110:46-50

6. Rasmussen BK (2001) Epidemiology of headache. Cephalalgia 21:774-777

7. Rasmussen BK (1995) Epidemiology of headache. Cephalalgia 15:45-68

8. Hale WE, May FE, Marks RG et al (1987) Headache in the elderly: an evaluation of risk factors. Headache 27:272-276

9. Srikiatkhachorn A (1991) Epidemiology of headache in the Thai elderly: a study in the Bangkae Home for the Aged. Headache 31:677-681

10. Franceschi M, Colombo B, Rossi P et al (1997) Headache in a population-based elderly cohort. An ancillary study to the Italian Longitudinal Study of Aging (ILSA). Headache 37:79-82

11. (2004) The international classification of headache disorders: 2 nd edn. Cephalalgia 24 (Suppl 1):9-160

12. Terwindt GM, Ferrari MD, Tijhuis M et al (2000) The impact of migraine on quality of life in the general population: the GEM study. Neurology 55:624-629

13. Elston Lafata J, Moon C, Leotta C et al (2004) The medical care utilization and costs associated with migraine headache. J Gen Intern Med 19:1005-1012

14. Lipton RB, Liberman JN, Kolodner KB et al (2003) Migraine headache disability and health-related quality-of-life: a population-based case-control study from England. Cephalalgia 23:441450

15. Tschannen TA, Duckro PN, Margolis RB et al (1992) The relationship of anger, depression, and perceived disability among headache patients. Headache 32:501-503

16. Jelinski SE, Magnusson JE, Becker WJ (2007) Factors associated with depression in patients referred to headache specialists. Neurology 68:489-495

17. Gesztelyi G, Bereczki D (2005) Disability is the major determinant of the severity of depressive symptoms in primary headaches but not in low back pain. Cephalalgia 25:598-604

18. Lanteri-Minet M, Radat F, Chautard MH et al (2005) Anxiety and depression associated with migraine: influence on migraine subjects disability and quality of life, and acute migraine management. Pain 118:319-326

19. Silberstein SD (2000) Practice parameter: evidence-based guidelines for migraine headache (an evidence-based review): report of the Quality Standards Subcommittee of the American Academy of Neurology. Neurology 55:754-762

20. Lipton RB, Stewart WF, Stone AM et al (2000) Stratified care versus step care strategies for migraine: the Disability in Strategies of Care (DISC) Study: a randomized trial. JAMA 284:2599-2605

21. Tepper SJ (2005) Comment on Igarashi Hisaka and Sakai Fumihiko. Current status of diagnosis and treatment of migraine headache in Japanese women. Headache Care. 2004, 1:207-213. Headache 45:184

22. Stewart WF, Lipton RB, Whyte J et al (1999) An international study to assess reliability of the Migraine Disability Assessment (MIDAS) score. Neurology 53:988-994

23. Stewart WF, Lipton RB, Kolodner K et al (1999) Reliability of the migraine disability assessment score in a population-based sample of headache sufferers. Cephalalgia 19:107-114 (discussion 174)

24. Ertas M, Siva A, Dalkara T et al (2004) Validity and reliability of the Turkish Migraine Disability Assessment (MIDAS) questionnaire. Headache 44:786-793

25. Gedikoglu U, Coskun O, Inan LE et al (2005) Validity and reliability of Turkish translation of Migraine Disability 
Assessment (MIDAS) questionnaire in patients with migraine. Cephalalgia 25:452-456

26. Gedikoglu U, Ucler S, Inan LE et al (2006) A preliminary study: validity and reliability of Turkish translation of migraine disability assessment (MIDAS) questionnaire in Turkish patients with chronic tension type headache. Int J Neurosci 116:1337-1345

27. Hung PH, Fuh JL, Wang SJ (2006) Validity, reliability and application of the Taiwan version of the migraine disability assessment questionnaire. J Formos Med Assoc 105:563-568

28. Iigaya M, Sakai F, Kolodner KB et al (2003) Reliability and validity of the Japanese Migraine Disability Assessment (MIDAS) Questionnaire. Headache 43:343-352

29. D'Amico D, Mosconi P, Genco S et al (2001) The Migraine Disability Assessment (MIDAS) questionnaire: translation and reliability of the Italian version. Cephalalgia 21:947-952

30. Pryse-Phillips W (2002) Evaluating migraine disability: the headache impact test instrument in context. Can J Neurol Sci 29(Suppl 2):S11-S15

31. Andrasik F, Lipchik GL, McCrory DC et al (2005) Outcome measurement in behavioral headache research: headache parameters and psychosocial outcomes. Headache 45:429-437

32. Gandek B, Alacoque J, Uzun V et al (2003) Translating the shortform Headache Impact Test (HIT-6) in 27 countries: methodological and conceptual issues. Qual Life Res 12:975-979

33. Kosinski M, Bayliss MS, Bjorner JB et al (2003) A six-item short-form survey for measuring headache impact: the HIT-6. Qual Life Res 12:963-974

34. Martin M, Blaisdell B, Kwong JW et al (2004) The short-form Headache Impact Test (HIT-6) was psychometrically equivalent in nine languages. J Clin Epidemiol 57:1271-1278

35. Jacobson GP, Ramadan NM, Aggarwal SK et al (1994) The Henry Ford Hospital Headache Disability Inventory (HDI). Neurology 44:837-842

36. Jacobson GP, Ramadan NM, Norris L et al (1995) Headache disability inventory (HDI): short-term test-retest reliability and spouse perceptions. Headache 35:534-539

37. Nunnally JC, Bernstein IH (1994) Psychometric theory. McGrawHill, New York

38. Beck AT, Weissman A, Lester D et al (1974) The measurement of pessimism: the hopelessness scale. J Consult Clin Psychol 42:861-865
39. Sheehan DV, Lecrubier Y, Sheehan KH et al (1998) The MiniInternational Neuropsychiatric Interview (M.I.N.I.): the development and validation of a structured diagnostic psychiatric interview for DSM-IV and ICD-10. J Clin Psychiatry 59(Suppl 20):22-33 quiz 34-57

40. Rutz W, von Knorring L, Pihlgren H et al (1995) Prevention of male suicides: lessons from Gotland study. Lancet 345:524

41. Rutz W (1999) Improvement of care for people suffering from depression: the need for comprehensive education. Int Clin Psychopharmacol 14(Suppl 3):S27-S33

42. Bedrosian RC, Beck AT (1979) Cognitive aspects of suicidal behavior. Suicide Life Threat Behav 9:87-96

43. Chandrasekaran R, Gnanaselane J (2005) Correlates of suicidal intent in attempted suicide. Hong Kong J Psychiatry 15:118-121

44. Wetzel RD, Margulies T, Davis R et al (1980) Hopelessness, depression, and suicide intent. J Clin Psychiatry 41:159-160

45. Beck AT, Steer RA, Kovacs M et al (1985) Hopelessness and eventual suicide: a 10-year prospective study of patients hospitalized with suicidal ideation. Am J Psychiatry 142:559-563

46. Beck AT, Brown G, Berchick RJ et al (1990) Relationship between hopelessness and ultimate suicide: a replication with psychiatric outpatients. Am J Psychiatry 147:190-195

47. Pompili M, Iliceto P, Lester D et al (2008) Beck Hopelessness Scale - manuale. Organizzazioni Speciali, Firenze

48. Zierau F, Bille A, Rutz W et al (2002) The Gotland Male Depression Scale: a validity study in patients with alcohol use disorder. Nord J Psychiatry 56:265-271

49. Abbate-Daga G, Fassino S, Lo Giudice R et al (2007) Anger, depression and personality dimensions in patients with migraine without aura. Psychother Psychosom 76:122-128

50. Silberstein SD, Lipton RB (1994) Overview of diagnosis and treatment of migraine. Neurology 44:S6-S16

51. Breslau N, Davis GC (1993) Migraine, physical health and psychiatric disorder: a prospective epidemiologic study in young adults. J Psychiatr Res 27:211-221

52. Breslau N (1992) Migraine, suicidal ideation, and suicide attempts. Neurology 42:392-395 